\title{
Dependência e resposta de mudas de cedro a fungos micorrízicos arbusculares
}

\author{
Fabiano Souza Rocha ${ }^{(1)}$, Orivaldo José Saggin Júnior(2), Eliane Maria Ribeiro da Silva( ${ }^{(2)}$ e Wallace Luís de Lima ${ }^{(1)}$ \\ (1)Universidade Federal Rural do Rio de Janeiro, BR 465, Km 07, CEP 23890-000 Seropédica, RJ. E-mail: fabinrural@zipmail.com.br, \\ limawl@yahoo.com.br (2)Embrapa Agrobiologia, BR 465, Km 07, Caixa Postal 74505, CEP 23890-000 Seropédica, RJ. E-mail: \\ saggin@cnpab.embrapa.br, eliane@cnpab.embrapa.br
}

Resumo - Os objetivos deste trabalho foram avaliar a resposta de mudas de cedro (Cedrela fissilis Vell.) a espécies de fungos micorrízicos arbusculares (FMA) e determinar o grau de dependência micorrízica dessas mudas. O ensaio foi conduzido em casa de vegetação, em delineamento de blocos inteiramente casualizados, em esquema fatorial 5 (doses de P) x 5 (4 espécies de FMA e testemunha sem inoculação), com seis repetições. Glomus clarum foi a espécie de FMA mais eficiente em promover o crescimento e a nutrição fosfatada de mudas de cedro, principalmente quando a inoculação foi realizada em substrato com P disponível em torno de $12 \mathrm{mg} \mathrm{dm}^{-3}$. A economia de $\mathrm{P}$ aplicado equivale a aproximadamente $63 \%$, em relação à testemunha sem inoculação, quando foram buscados $80 \%$ do crescimento máximo. Verificou-se elevado grau de dependência micorrízica das mudas de cedro e, portanto, a inoculação de FMA é um procedimento que deve ser considerado na fase de viveiro, para garantir o sucesso do estabelecimento e crescimento em campo dessas mudas.

Termos para indexação: Cedrela fissilis, endossimbiose, nutrição fosfatada, eficiência simbiótica, fósforo, inoculação.

\section{Cedar seedlings dependency and responsiveness to arbuscular mycorrhizal fungi}

\begin{abstract}
The objectives of this work were to evaluate cedar (Cedrela fissilis Vell.) seedlings responsiveness to arbuscular mycorrhizal fungi (AMF) species and to determine mycorrhizal dependency degree of this seedling. The experiment was carried out at greenhouse, in a complete randomized block design as a factorial set 5 (P doses) x 5 (4 AMF species + noninoculated treatment) with six replications. Glomus clarum was the most efficient AMF species in promoting the growth and P nutrition of cedar seedlings, especially when the inoculation was done in substratum containing $\mathrm{P}$ available levels close to $12 \mathrm{mg} \mathrm{dm}^{-3}$. The saving of applied $\mathrm{P}$ was approximately $63 \%$, regarding noninoculated treatment when $80 \%$ of the maximum growth was estimated. The elevated degree of mycorrhizal dependency of cedar seedlings was verified, so AMF inoculation is a procedure that should be considered in nursery stage, in order to guarantee cedar seedlings successful establishment and growth on field conditions.
\end{abstract}

Index terms: Cedrela fissilis, endosymbiosis, P nutrition, symbiotic effectiveness, phosphorus, inoculation.

\section{Introdução}

A resposta das plantas às micorrizas varia nas diferentes combinações fungo/planta/ambiente (Saggin Júnior et al., 1994), e pode ser avaliada pelo crescimento e produtividade da planta, já que essa é o simbionte de maior interesse econômico. Diferentes espécies de fungos micorrízicos arbusculares (FMA) devem ser testadas em uma mesma planta, sob as mesmas condições ambientais, para selecionar FMA eficientes quanto à capacidade de promover o crescimento de seu hospedeiro (Paula et al., 1990; Saggin Júnior \& Siqueira, 1995).
Em espécies florestais, depois da seleção de espécies de FMA eficientes em promover crescimento, o sucesso e a viabilidade de sua aplicação no estágio da formação de mudas dependerá do grau de micotrofismo, ou seja, da dependência que a planta apresenta a essa simbiose (Siqueira \& Saggin Júnior, 2001). Além disso, do grau de micotrofismo da espécie arbórea depende o sucesso de seu estabelecimento no campo, quando na área não houver propágulos micorrízicos ou disponibilidade de nutrientes, em especial o fósforo (Janos, 1987). Assim, é importante conhecer a dependência micorrízica das espécies arbóreas, uma vez que a sobrevivência e 
competição dessas são alteradas pelo seu micotrofismo (Pouyú-Rojas, 2002), que altera a dinâmica das populações e seu papel nas comunidades vegetais (Janos, 1988), havendo, às vezes, necessidade de se inocular FMA para o crescimento de mudas (Siqueira \& Saggin Júnior, 2001).

O cedro (Cedrela fissilis Vell.) é uma espécie com elevada dependência micorrízica (Saggin Júnior, 1997). Como tem ampla dispersão territorial, sendo encontrada em distintos biomas brasileiros (Lorenzi, 2000), podem ocorrer diferentes ecótipos dessa planta, quanto ao grau de dependência micorrízica, já que essa característica genética varia até entre variedades e cultivares (Hetrick et al., 1993; Trindade et al., 2001). Também existe a necessidade de se avaliar a resposta de cedro a diferentes FMA para seleção de combinações eficientes.

Os objetivos deste trabalho foram avaliar a resposta de mudas de cedro (Cedrela fissilis Vell.) a espécies de FMA e determinar o grau de dependência micorrízica dessas mudas.

\section{Material e Métodos}

Em abril de 2003, instalou-se um experimento em casa de vegetação da Embrapa Agrobiologia, Seropédica, RJ, em tubetes com $1 \mathrm{~kg}$ do horizonte B de um Argissolo Vermelho-Amarelo argiloso distrófico que, depois de autoclavado, apresentou as seguintes características químicas: pH em água (1:2,5), 5; P e K extraídos por Mehlich-1, 2 e $52 \mathrm{mg} \mathrm{dm}^{-3}$, respectivamente; $\mathrm{Al}$, Ca e Mg trocáveis ( $\mathrm{KCl} 1 \mathrm{~mol} \mathrm{~L}^{-1}$ ), 0,2, 1,7 e $1 \mathrm{cmol}_{\mathrm{C}} \mathrm{dm}^{-3}$, respectivamente.

O experimento foi instalado em delineamento de blocos ao acaso, em esquema fatorial $5 \times 5$, com seis repetições. Os tratamentos corresponderam a cinco doses de superfosfato triplo moído, combinadas com quatro espécies de FMA e uma testemunha sem inoculação. As doses de $\mathrm{P}$ foram estabelecidas com base no $\mathrm{P}$ remanescente (Alvarez et al., 2000), com 0, 30, 240, 480 e $960 \mathrm{mg} \mathrm{kg}^{-1}$ de $\mathrm{P}$ aplicados no solo, que resultaram em 7, 9, 34, 79 e $164 \mathrm{mg} \mathrm{dm}^{-3}$ de $\mathrm{P}$ disponível pelo extrator Mehlich-1, respectivamente. A relação linear entre $\mathrm{P}$ aplicado e $\mathrm{P}$ disponível no solo foi de $\mathrm{y}=1,980+0,1655 \mathrm{x}\left(\mathrm{R}^{2}=0,99\right)$.

As espécies de FMA utilizadas foram Glomus clarum Nicolson \& Schenck, Gigaspora margarita Becker \& Hall, Glomus etunicatum Becker \& Gerdemann e Scutellospora heterogama (Nicol \& Gerd) Walker \& Sanders. Cada recipiente recebeu 5 g de solo-inóculo de um dos quatro FMA, com cer- ca de 160 esporos. Para tentar uniformizar os tratamentos quanto aos demais componentes da microbiota do inóculo, adicionou-se, em todos os recipientes, $10 \mathrm{~mL}$ de um filtrado isento de propágulos de FMA, preparado com os solos-inóculos dos quatro FMA testados.

Sementes de cedro, cedidas pelo Instituto de Pesquisas e Estudos Florestais (IPEF), foram desinfestadas superficialmente (álcool 70\%-1 minuto; hipoclorito de sódio $2 \%-3$ minutos) e semeadas diretamente, três por recipiente. Vinte dias após a germinação, as plântulas foram desbastadas, tendo-se deixado apenas uma muda por recipiente. Cada muda recebeu, semanalmente, $10 \mathrm{~mL}$ de solução nutritiva com $\left(\mathrm{mg} \mathrm{L}^{-1}\right)$ : $\mathrm{Ca}\left(\mathrm{NO}_{3}\right)_{2} \cdot 4 \mathrm{H}_{2} \mathrm{O}, 0,354 ; \mathrm{MgSO}_{4} \cdot 7 \mathrm{H}_{2} \mathrm{O}, 0,148 ; \mathrm{KNO}_{3}$, 0,$152 ; \mathrm{ZnSO}_{4} \cdot 7 \mathrm{H}_{2} \mathrm{O}, 0,06 ; \mathrm{H}_{3} \mathrm{BO}_{3}, 0,86 ; \mathrm{CuSO}_{4} \cdot 5 \mathrm{H}_{2} \mathrm{O}$, 0,24; $\mathrm{NaMoO}_{4} \cdot 2 \mathrm{H}_{2} \mathrm{O}, 0,005$.

Foram feitas quatro avaliações durante a condução do experimento. Na primeira, aos 45 dias após a emergência das plântulas (DAE), avaliaram-se altura e diâmetro do colo. Aos 90 e 120 DAE, avaliaram-se altura, diâmetro do colo e quantidade de $\mathrm{P}$ em discos de folhas de $0,5 \mathrm{~cm}^{2}$ (Aziz \& Habte, 1987). Na última avaliação, aos 180 DAE, avaliaram-se altura, diâmetro do colo, massa de raízes e parte aérea frescas e secadas em estufa a $65^{\circ} \mathrm{C}$. Nessa ocasião avaliou-se a colonização radicular, em amostras de $1 \mathrm{~g}$ de raízes frescas, após clarificação e coloração (Koske \& Gemma, 1989; Grace \& Stribley, 1991).

Os dados, testados quanto à sua homogeneidade de variância, foram submetidos à análise de variância e teste de média (Scott-Knott 5\%) e regressão polinomial, utilizando-se o programa estatístico Sisvar (Ferreira, 1998).

Para cada FMA foram calculadas a faixa de mutualismo, o benefício do fósforo, o benefício micorrízico e a eficiência simbiótica, conforme Saggin Júnior \& Siqueira (1995), utilizando-se as curvas de resposta ao $\mathrm{P}$ aplicado, estimadas por regressão polinomial.

A faixa de mutualismo é dada pelas doses de $\mathrm{P}$ aplicadas ao solo, em que as curvas das plantas com inoculação sem inoculação se cruzam.

O benefício do fósforo foi calculado pela integral da curva das plantas sem inoculação, definida entre as doses mínima e máxima de $\mathrm{P}$ aplicado ao solo.

O benefício micorrízico foi calculado pela integral da curva das plantas com inoculação definida pela faixa de mutualismo, menos a integral da curva das plantas sem inoculação definida pela faixa de mutualismo. 
A eficiência simbiótica foi calculada pela relação: 100 x (benefício micorrízico/benefício do fósforo).

Utilizando-se as curvas de resposta ao $\mathrm{P}$ da parte aérea seca, calculou-se também a resposta máxima de cada FMA em relação à testemunha sem inoculação, e a dose de $\mathrm{P}$ em que cada FMA promoveu um crescimento relativo a $80 \%$ da estimativa máxima de crescimento, considerada, nesse caso, a da testemunha sem inoculação na dose $960 \mathrm{mg} \mathrm{kg}^{-1}$.

\section{Resultados e Discussão}

Na análise dos dados de altura das plantas, os benefícios da inoculação, particularmente com G. margarita, iniciaram-se, aparentemente, a partir dos 45 dias após a emergência (DAE) das sementes. A análise estatística utilizada indicou efeito significativo dessa espécie somente quando as mudas já estavam formadas (180 DAE), quando a espécie G. clarum se destacou, com G. margarita, dos demais tratamentos (Figura 1).
Algumas espécies de FMA possuem maior capacidade de se estabelecerem e beneficiarem a planta hospedeira, em relação a outras menos eficientes (Abbott \& Robson, 1981). Saggin Júnior \& Siqueira (1995) sugerem que tais diferenças são causadas pelo balanço entre o benefício nutricional, promovido pelo fungo à planta, e o dreno de fotoassimilados da planta para o fungo. Observa-se que esse delicado balanço tem forte interação com o teor de $\mathrm{P}$ disponível no substrato. Assim, é possível que mesmo um fungo eficiente possa promover um grande dreno de fotossintatos, durante a fase de maior aumento na colonização das raízes (Siqueira et al., 1994). Isso poderia justificar o fato de G. margarita e, particularmente, de G. clarum, apresentarem benefícios ao crescimento do cedro significativos apenas aos 180 DAE.

Em relação ao diâmetro do colo, não foram verificados efeitos significativos dos FMA, o que corrobora os dados de Carneiro et al. (1996), também com cedro, e isto foi observado em todas as épocas avaliadas. Aos

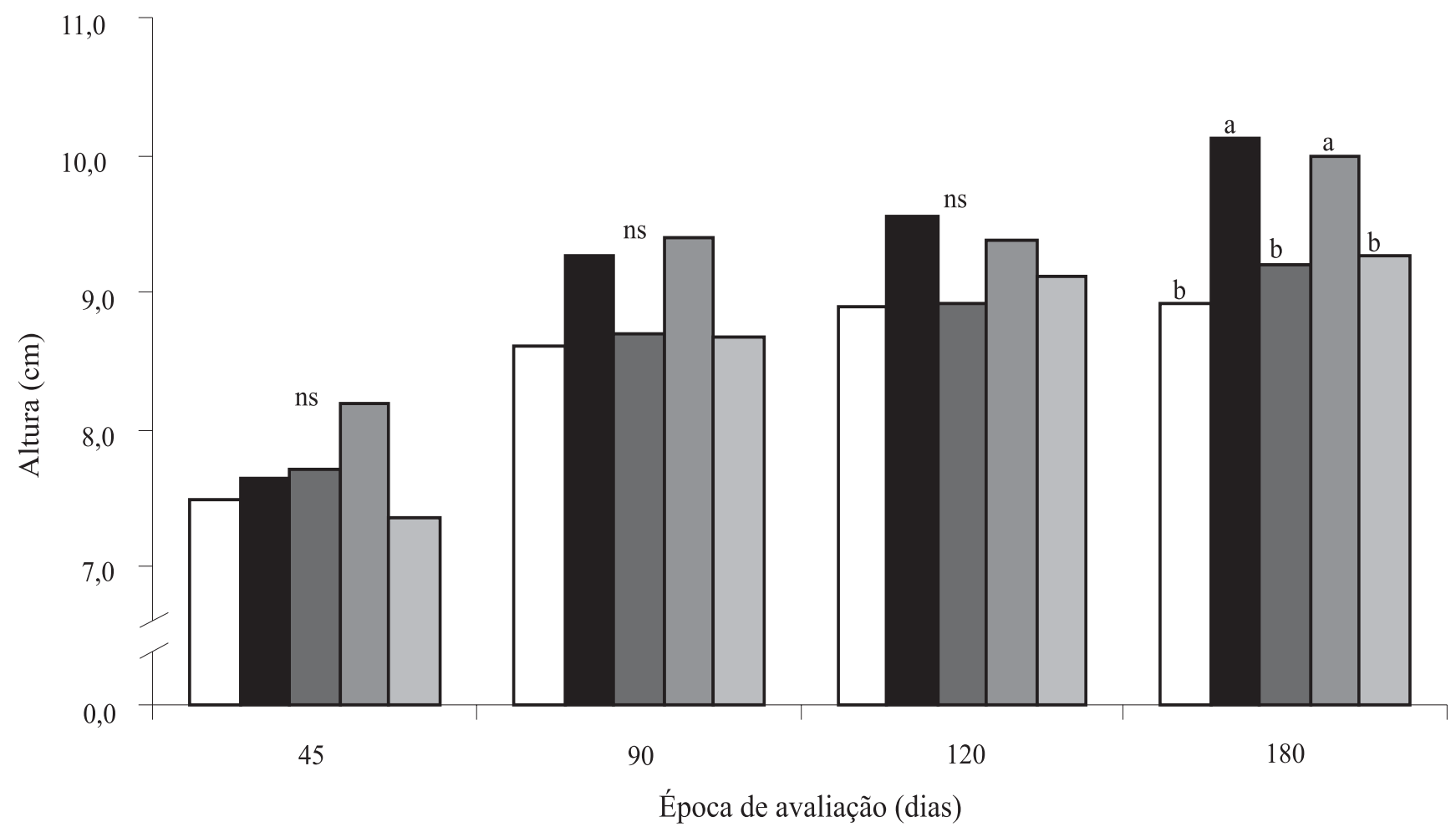

$\square_{\text {Controle }} \square_{\text {G. clarum }} \square_{\text {G. etunicatum }} \quad \square_{\text {G. margarita }} \quad \square$ S. heterogama

Figura 1. Altura de mudas de cedro com inoculação de diferentes espécies de fungos micorrízicos arbusculares, em diferentes

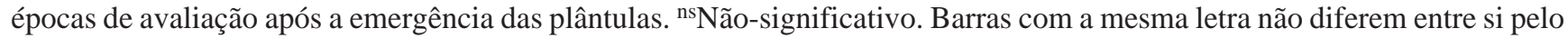
teste de Scott-Knott a 5\% de probabilidade. 
180 DAE, o diâmetro do colo variou de 5,9 mm, nas plantas com inóculo de G. etunicatum, até 6,7 mm naquelas com inóculo de G. clarum.

A partir dos 120 DAE, as variáveis foram influenciadas pelas doses de $\mathrm{P}$, com efeitos diferenciados para cada fungo, na maioria delas. Aos 180 DAE, para altura e massa da matéria seca da parte aérea, as plantas associadas a fungos micorrízicos apresentaram respostas quadrática e raiz-quadrada, enquanto aquelas sem inoculação apresentaram respostas lineares (Figura 2). Ajustes quadrático e raiz-quadrada são típicos de respostas de plantas associadas a fungos micorrízicos ao $\mathrm{P}$ (Saggin Júnior \& Siqueira, 1996; Carneiro et al., 1999; Trindade et al., 2001). Esse comportamento está associado ao fato de os FMA promoverem maiores benefícios com baixa disponibilidade de $\mathrm{P}$, efeito que é diminuído com a maior disponibilidade desse nutriente, chegando ao ponto de o fungo ser desnecessário à planta. Isto foi verificado em várias espécies florestais por Siqueira \& Saggin Júnior (2001).

As mudas com inoculação apresentaram resposta máxima em crescimento, entre as doses 30 e $480 \mathrm{mg} \mathrm{kg}^{-1}$, e redução no crescimento na dose $960 \mathrm{mg} \mathrm{kg}^{-1}$ (Figura 2). Isto evidencia que, com o aumento do $\mathrm{P}$ aplicado ao solo, os FMA passam a ser desnecessários à planta e a representar um gasto fotossintético (Siqueira \& Colozzi Filho, 1986). Esse comportamento foi verificado em todas as variáveis de crescimento. Entretanto, plantas com inoculação da maioria dos FMA apresentaram aumento linear das quantidades de $\mathrm{P}$ nos discos de folhas, em resposta à adição de $\mathrm{P}$ ao solo (Figura 3). Isto indica que nas maiores doses existia acúmulo de $\mathrm{P}$ na parte aérea, sem correspondente crescimento vegetativo, o que sugere um consumo de luxo de P (Stribley et al., 1980; Bolan, 1991).

Com base nos dados de matéria seca da parte aérea (Figura 2), calculou-se a resposta máxima a cada FMA, a fim de se determinar a dose ótima de $\mathrm{P}$ para promover resposta da inoculação no crescimento de cedro (Tabela 1). A resposta máxima de G. clarum foi pelo menos o dobro das calculadas para as demais espécies de FMA, e foi obtida em uma dose de $P$ cinco vezes menor do que as doses de resposta máxima dos demais FMA, o que indica maior eficiência de G. clarum em promover o crescimento de mudas de cedro. Saggin Júnior \& Silva (2002), ao revisarem valores de P disponível no solo, em que foram observadas respostas máximas à inoculação de FMA em diferentes plantas, verificaram que tais valores variam de 9 a $27 \mathrm{mg} \mathrm{dm}^{-3}$. Assim, o valor de $12,2 \mathrm{mg} \mathrm{dm}^{-3}$, estimado como ótimo neste trabalho, para cedro inoculado com G. clarum, está dentro dessa faixa, e um nível de $\mathrm{P}$ disponível em torno desse valor irá refletir no melhor desempenho desse fungo em promover o crescimento de mudas de cedro. Entretanto, a dose de $\mathrm{P}$ de melhor desempenho dos FMA não reflete sempre o maior crescimento da planta, como pode ser observado pela matéria seca da parte aérea na resposta máxima (Tabela 1). Assim, é importante avaliar a eficiência dos FMA, quando a planta obtém um crescimento satisfatório, como com $80 \%$ do crescimento máximo.
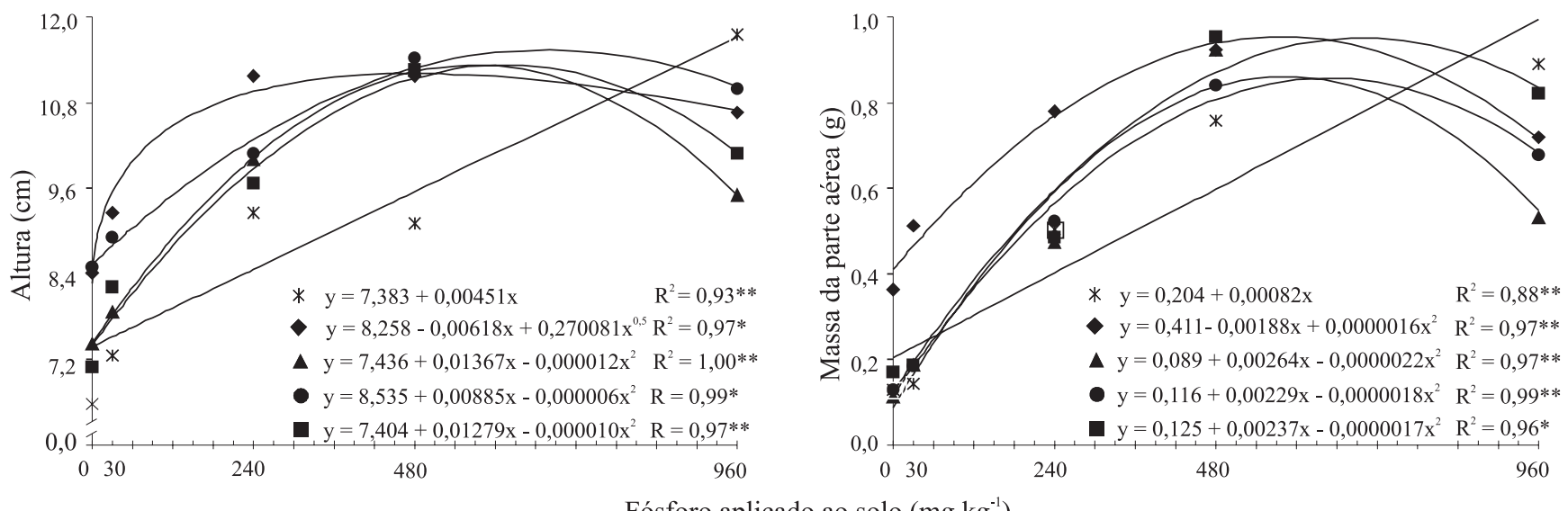

Fósforo aplicado ao solo $\left(\mathrm{mg} \mathrm{kg}^{-1}\right)$

Figura 2. Altura e massa de matéria seca da parte aérea de mudas de cedro sem inoculação ( $*$ ) e com inoculação dos fungos micorrízicos arbusculares: Glomus clarum $(\bullet)$, Glomus etunicatum (A), Gigaspora margarita (O) e Scutellospora heterogama ( $\square$ ), em função do P aplicado ao solo, aos 180 dias após a emergência das plântulas. * e **Significativo a 5 e $1 \%$, de probabilidade, respectivamente, pelo teste $\mathrm{F}$. 
A eficiência do G. clarum em beneficiar o cedro em doses menores de $\mathrm{P}$, comparado aos demais FMA, também foi evidenciada, quando se calculou a dose de P no solo responsável por $80 \%$ do crescimento máximo. G. clarum promoveu 80\% do máximo com $266 \mathrm{mg} \mathrm{kg}^{-1}$, enquanto G. etunicatum com $414 \mathrm{mg} \mathrm{kg}^{-1}$, G. margarita com $459 \mathrm{mg} \mathrm{kg}^{-1} \mathrm{e}$ S. heterogama com $395 \mathrm{mg} \mathrm{kg}^{-1}$. Ou seja, uma dose de P 37\% menor do que a média desses três últimos fungos, e $63 \%$ menor que $719 \mathrm{mg} \mathrm{kg}^{-1}$, em que ocorreu $80 \%$ do crescimento máximo da testemunha sem inoculação. O benefício de G. clarum à planta hospedeira parece estar relacionado com sua maior eficiência em absorver P do solo e mantêlo em altos níveis nos tecidos da planta, como observado pela quantidade de $\mathrm{P}$ nos discos de folha aos $120 \mathrm{DAE}$ (Figura 3). A maior absorção de P por plantas associadas a fungos micorrízicos tem sido atribuída à maior exploração e contato com o solo e à maior eficiência das hifas na absorção (Bolan, 1991).

G. clarum promoveu os maiores valores de colonização micorrízica nas raízes do cedro, na maioria das doses de $\mathrm{P}$, e mostrou-se superior aos demais FMA nas doses 30, 480 e $960 \mathrm{mg} \mathrm{kg}^{-1}$ de P (Tabela 2). Não houve efeito das doses de $P$ sobre a colonização micorrízica em todos os FMA testados.

A eficiência de G. clarum em promover o crescimento de mudas de cedro foi quantificada conforme Saggin Júnior \& Siqueira (1995). G. clarum apresenta maior eficiência que os demais FMA(Tabela 3). A magnitude da eficiência simbiótica pode diferir entre os FMA testados e entre as variáveis consideradas (Sieverding, 1991; Saggin Júnior \& Siqueira, 1995). Neste trabalho, quando baseada na matéria seca da parte aérea, a eficiência simbiótica foi maior do que na altura das mudas e
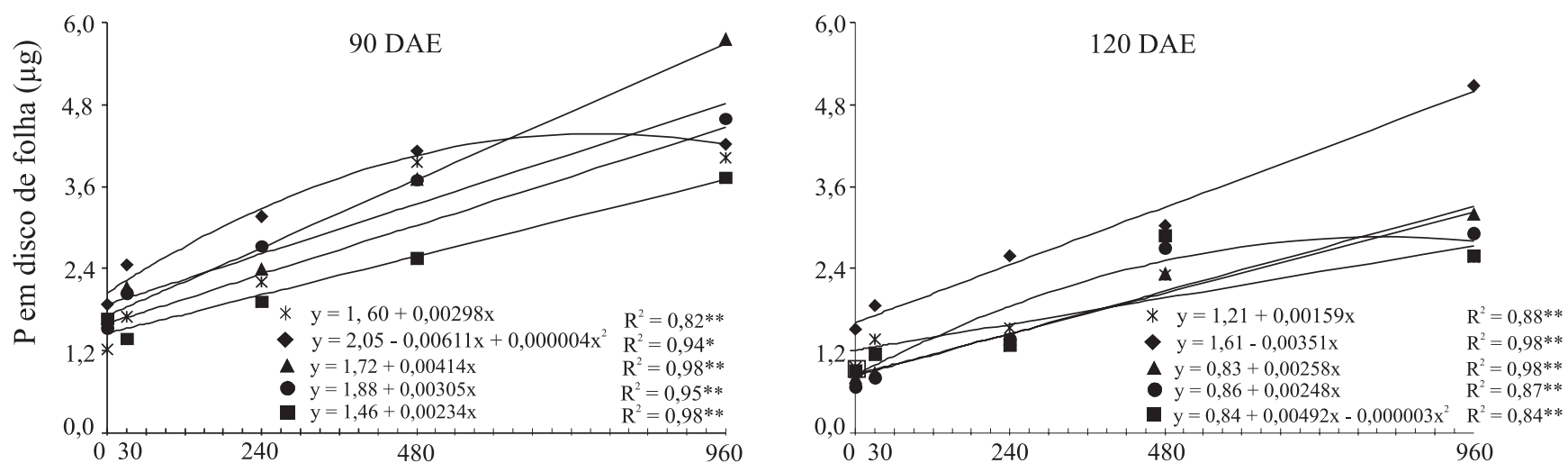

Fósforo aplicado ao solo $\left(\mathrm{mg} \mathrm{kg}^{-1}\right)$

Figura 3. Quantidade de P em discos de folhas de $0,5 \mathrm{~cm}^{2}$, aos 90 e 120 dias após a emergência (DAE) de mudas de cedro sem inoculação (*) e com inoculação dos fungos micorrízicos arbusculares: Glomus clarum ( $\bullet$ ), Glomus etunicatum (A), Gigaspora margarita ( ) e Scutellospora heterogama $(\square)$, em função do P aplicado ao solo. * e **Significativo a 5 e $1 \%$, de probabilidade, respectivamente, pelo teste $\mathrm{F}$.

Tabela 1. Resposta máxima em relação à testemunha sem inoculação, de mudas de cedro com inoculação de diferentes espécies de fungos micorrízicos arbusculares, dose de $\mathrm{P}$ da resposta máxima, respectivo $\mathrm{P}$ disponível no solo e massa de matéria seca da parte aérea (MMSPA) obtida.

\begin{tabular}{|c|c|c|c|c|}
\hline Espécies & $\begin{array}{l}\text { Resposta máxima em relação } \\
\text { à testemunha }(\%)^{(1)}\end{array}$ & $\begin{array}{l}\text { Dose de } \mathrm{P} \text { da resposta } \\
\text { máxima }\left(\mathrm{mg} \mathrm{kg}^{-1}\right)^{(2)}\end{array}$ & $\begin{array}{l}\text { P disponível } \\
\left(\mathrm{mg} \mathrm{dm}^{-3}\right)^{(3)}\end{array}$ & $\begin{array}{c}\text { MMSPA na resposta } \\
\text { máxima }(\mathrm{g})\end{array}$ \\
\hline Glomus clarum & 104 & 62 & 12,2 & 0,52 \\
\hline Glomus etunicatum & 50 & 312 & 53,6 & 0,69 \\
\hline Glomus margarita & 43 & 315 & 54,1 & 0,66 \\
\hline Scutellospora heterogama & 52 & 330 & 56,4 & 0,72 \\
\hline
\end{tabular}

(1)Equivalente ao valor R de Janos (1988), calculado pelas curvas de resposta ao P obtidas com a massa de matéria seca da parte aérea. (2)Equivalente à dose de $\mathrm{P}$ aplicada ao solo. ${ }^{(3)}$ Estimado pela relação linear entre $\mathrm{P}$ aplicado e $\mathrm{P}$ disponível no solo (extraído por meio de Mehlich-1). 
gerou diferenças entre os FMA, pois G. margarita foi mais eficiente do que G. etunicatum e $S$. heterogama, em promover o crescimento em altura; no entanto, apresentou menor eficiência que esses fungos, em promover o aumento da massa de matéria seca da parte aérea. G. clarum apresentou maior eficiência em ambas as variáveis, e apresentou benefício micorrízico na massa de matéria seca da parte aérea até a dose de $808 \mathrm{mg} \mathrm{kg}^{-1}$ (Tabela 3), correspondente a $136 \mathrm{mg} \mathrm{dm}^{-3}$ de P disponível, tendo demonstrado benefício mesmo em solos com alto teor de P disponível.

Segundo Saggin Júnior \& Siqueira (1995), os fungos mais eficientes são aqueles que conseguem manter maior benefício à planta, sob maior gradiente de $\mathrm{P}$ disponível no solo, denominado de faixa de mutualismo. A faixa de mutualismo é compreendida pelos níveis de $\mathrm{P}$ onde a simbiose mantém o benefício à planta e, segundo esses autores, apresenta relação direta com a eficiência do fungo.

Não se observou relação direta entre a faixa de mutualismo e a eficiência simbiótica dos FMA. O limite inferior da faixa de mutualismo, em termos de P disponível, ficou entre 2 e $14 \mathrm{mg} \mathrm{dm}^{-3}$, e o limite superior entre 124 e $145 \mathrm{mg} \mathrm{dm}^{-3}$, variando com a espécie de fungo e com a variável considerada (Tabela 3). Esses resultados corroboram os de Siqueira \& ColozziFilho (1986) para a faixa de transição do caráter da simbiose de prejudicial à benéfica, com valores entre 3 e $10 \mathrm{mg} \mathrm{dm}^{-3}$ e entre 100 e $300 \mathrm{mg} \mathrm{dm}^{-3}$ de P disponível no solo (extraído por Mehlich-1). A adaptação do FMA a diferentes condições de P disponível é importante quando se tratam mudas, que geralmente são produzidas em substratos com elevada disponibilidade de P e, posteriormente, são transplantadas para solos com menor fertilidade.

Pouýu Rojas (2002), ao trabalhar com 16 espécies florestais de distintos grupos sucessionais com inoculação

Tabela 2. Colonização micorrízica (\%) das raízes de mudas de cedro com inoculação de diferentes fungos micorrízicos arbusculares, aos 180 dias após a emergência das plântulas, com diferentes doses de fósforo ${ }^{(1)}$.

\begin{tabular}{lrrrrrr}
\hline Espécies & \multicolumn{5}{c}{ Dose de $\mathrm{P}\left(\mathrm{mg} \mathrm{kg}^{-1}\right)$} & \multirow{2}{*}{ Média } \\
\cline { 2 - 6 } & 0 & \multicolumn{1}{c}{30} & 240 & 480 & 960 & \\
\hline Testemunha & $0,0 \mathrm{~b}$ & $0,0 \mathrm{~b}$ & $0,0 \mathrm{~b}$ & $0,0 \mathrm{~b}$ & $0,0 \mathrm{~b}$ & $0,0 \mathrm{c}$ \\
Glomus clarum & $14,3 \mathrm{a}$ & $22,2 \mathrm{a}$ & $17,1 \mathrm{a}$ & $30,5 \mathrm{a}$ & $19,8 \mathrm{a}$ & $20,8 \mathrm{a}$ \\
Glomus etunicatum & $15,4 \mathrm{a}$ & $8,4 \mathrm{~b}$ & $12,8 \mathrm{a}$ & $7,8 \mathrm{~b}$ & $8,4 \mathrm{~b}$ & $10,6 \mathrm{~b}$ \\
Glomus margarita & $12,3 \mathrm{a}$ & $9,9 \mathrm{~b}$ & $13,3 \mathrm{a}$ & $2,4 \mathrm{~b}$ & $7,2 \mathrm{~b}$ & $9,0 \mathrm{~b}$ \\
Scutellospora heterogama $10,1 \mathrm{a}$ & $8,8 \mathrm{~b}$ & $13,0 \mathrm{a}$ & $9,3 \mathrm{~b}$ & $6,3 \mathrm{~b}$ & $9,5 \mathrm{~b}$ \\
\hline
\end{tabular}

(1)Médias seguidas de mesma letra, na coluna, não diferem entre si pelo teste de Scott-Knott a 5\% de probabilidade. de diferentes FMA, também constatou alta capacidade de $G$. clarum em beneficiar mudas de cedro. O mesmo autor verificou que esse fungo foi capaz de promover o crescimento da maioria das espécies arbóreas em que foi inoculado. G. clarum também foi o mais eficiente em promover o crescimento de Mimosa artemisiana (Sibinel, 2003), que tem grande potencial para revegetação de áreas degradadas na Mata Atlântica, e mostrou benefício em mudas de laranja, tangerina (Melloni \& Cardoso, 1999) e seringueira (Lopes \& Cardoso, 2001). A capacidade de uma espécie de FMA em beneficiar uma ampla variedade de plantas é relevante na seleção de fungos para programas de inoculação.

A fim de minimizar o fato de um FMA apresentar diferentes magnitudes de eficiência simbiótica, de acordo com a variável analisada, os FMA testados foram enquadrados em categorias de eficiência baseados em mais de uma variável, conforme proposto por Saggin Júnior \& Siqueira (1995), tendo-se obtido a média da eficiência simbiótica com a altura e matéria seca da parte aérea (Tabela 3). Assim, G. clarum foi categorizado como de alta eficiência em promover o crescimento do cedro, e os demais classificados como de baixa eficiência. Essa categorização é relativa e se baseia na comparação entre os FMA testados, o que não significa que um dos FMA categorizados como de baixa eficiência não seja o mais eficiente, em comparação a outros fungos não testados aqui, ou em outra situação com outras espécies vegetais.

De acordo com a quantificação de dependência micorrízica de Janos (1988), quanto maior for o nível de

Tabela 3. Faixa de mutualismo, benefício micorrízico (BM), benefício do fósforo (BF) e eficiência simbiótica (ES) de diferentes espécies de fungos micorrízicos arbusculares, baseada na altura e na massa de matéria seca da parte aérea do cedro aos 180 dias após emergência das plântulas.

\begin{tabular}{|c|c|c|c|c|c|}
\hline \multirow[t]{2}{*}{ Espécie } & \multicolumn{2}{|c|}{ Faixa de mutualismo } & BM & $\mathrm{BF}$ & \multirow{2}{*}{$\begin{array}{l}\text { ES } \\
(\%)\end{array}$} \\
\hline & $\begin{array}{c}\mathrm{P} \text { aplicado } \\
\left(\mathrm{mg} \mathrm{kg}^{-1}\right)\end{array}$ & $\begin{array}{l}\text { P disponível }{ }^{(2)} \\
\left(\mathrm{mg} \mathrm{dm}^{-3}\right)\end{array}$ & \multicolumn{2}{|c|}{------ (área) $)^{(3)}$------ } & \\
\hline & \multicolumn{4}{|c|}{ Altura } & \\
\hline Glomus clarum & $0-793$ & $2-133$ & 1.352 & 9.166 & 15 \\
\hline Glomus etunicatum & $0-769$ & $2-129$ & 930 & 9.166 & 10 \\
\hline Glomus margarita & $0-869$ & $2-145$ & 1.213 & 9.166 & 13 \\
\hline \multirow[t]{2}{*}{ Scutellospora heterogama } & $0-799$ & $2-134$ & 891 & 9.166 & 10 \\
\hline & \multicolumn{5}{|c|}{ Massa da parte aérea seca } \\
\hline Glomus clarum & $0-808$ & $2-136$ & 226 & 575 & 39 \\
\hline Glomus etunicatum & $70-737$ & $14-124$ & 107 & 575 & 19 \\
\hline Gigaspora margarita & $65-765$ & $13-129$ & 97 & 575 & 17 \\
\hline Scutellospora heterogama & $55-857$ & $11-144$ & 133 & 575 & 23 \\
\hline
\end{tabular}

(1)Equivalente à dose de $\mathrm{P}$ aplicada ao solo. ${ }^{(2)}$ Estimado pela relação linear entre $\mathrm{P}$ aplicado e $\mathrm{P}$ disponível no solo (extraído por meio de Mehlich-1). ${ }^{(3)}$ Estimada por cálculo de integral definida pela faixa de mutualismo. 
P na solução do solo, em que a inoculação de FMA mantém benefícios à planta, maior será a dependência da planta das micorrizas. Esse teor de P foi denominado pelo autor como T' e representa o teor de $\mathrm{P}$ no solo necessário para substituir a micorriza.

No presente trabalho, T' foi calculado com base no valor médio do limite superior da faixa de mutualismo, obtida pelos diferentes FMA testados nas duas variáveis analisadas (Tabela 3). Esse valor foi igual a $800 \mathrm{mg} \mathrm{kg}^{-1}$ de $\mathrm{P}$ aplicado ao solo, ou equivalente a $134,4 \mathrm{mg} \mathrm{dm}^{-3}$ de P disponível no solo extraído por Mehlich-1.

Embora esses valores não sejam comparáveis ao valor de T' do cedro $\left(0,297 \mathrm{mg} \mathrm{L}^{-1}\right.$ de P na solução do solo), obtido por Siqueira \& Saggin-Júnior (2001), quando usaram a variável original da quantificação de Janos (1988), pode concluir, pelo nível de P necessário para substituir a micorriza, que os resultados deste trabalho corroboram os de Siqueira \& Saggin Junior (2001), e demonstram que o cedro apresenta elevada dependência das micorrizas. Isso porque o valor de $0,297 \mathrm{mg} \mathrm{L}^{-1} \mathrm{de}$ $\mathrm{P}$ na solução do solo é considerado alto, quase $50 \%$ acima do nível ótimo para nutrição de plantas (Fox \& Kamprath, 1970), e o valor de $134,4 \mathrm{mg} \mathrm{dm}^{-3}$ de P disponível no solo, extraído por Mehlich-I, também é considerado alto (CFSEMG, 1999).

Verificou-se, assim, elevado grau de dependência micorrízica das mudas de cedro e, portanto, a inoculação de FMA é um procedimento que deve ser considerado na fase de viveiro, para garantir o sucesso do estabelecimento e crescimento em campo dessas mudas.

\section{Conclusões}

1. Entre os fungos micorrízicos arbusculares testados, G. clarum é o mais eficiente em promover o crescimento e nutrição fosfatada do cedro.

2. A eficiência de G. clarum em promover o crescimento de mudas de cedro é aumentada quando a inoculação é feita em substrato com níveis de $\mathrm{P}$ disponível em torno de $12 \mathrm{mg} \mathrm{dm}^{-3}$.

3. A economia na fertilização fosfatada da muda de cedro, promovida por G. clarum é de $37 \%$ em relação aos demais fungos micorrízicos arbusculares, e de 63\% em relação à testemunha sem inoculação.

\section{Referências}

ABBOTT, L.K.; ROBSON, A.D. Infectivity and effectiveness of five endomycorrhizal fungi: competition with indigenous fungi in field soils. Australian Journal of Agricultural Research, v.32, p.621-630, 1981.

ALVAREZ, V.H.; NOVAIS, R.F.; DIAS, L.E.; OLIVEIRA, J.A. Determinação e uso do fósforo remanescente. Viçosa: Sociedade Brasileira de Ciência do Solo, 2000. v.25, p.27-33.

AZIZ, T.; HABTE, M. Determining vesicular-arbuscular mycorrhizal effectiveness by monitoring $\mathrm{P}$ status of leaf disks. Canadian Journal of Microbiology, v.33, p.1097-1101, 1987.

BOLAN, N.S. A critical review on the role of mycorrhizal fungi in the uptake of phosphorus by plants. Plant and Soil, v.134, p.189207, 1991.

CARNEIRO, M.A.C.; SIQUEIRA, J.O.; CURI, N.; MOREIRA, F.M.S. Efeitos da inoculação de fungos micorrízicos arbusculares e da aplicação de fósforo no estabelecimento de forrageiras em solo degradado. Pesquisa Agropecuária Brasileira, v.34, p.1669-1677, 1999.

CARNEIRO, M.A.C.; SIQUEIRA, J.O.; DAVIDE, A.C.; GOMES, L.J.; CURI, N.; VALE, F.R. do. Fungos micorrízicos e superfosfato no crescimento de espécies arbóreas tropicais. Scientia Forestalis, v.50, p.21-36, 1996.

CFSEMG. Recomendação para o uso de corretivos e fertilizantes em Minas Gerais. 5a aproximação. Viçosa, 1999. 359p.

FERREIRA, D.F. Sistemas de análise estatística para dados balanceados. Lavras: Ufla: DEX: Sisvar, 1998. 141p.

FOX, R.L.; KAMPRATH, E.J. Phosphate sorption isotherms for evaluating the phosphate requirements of soils. Soil Science Society of America Proceedings, v.34, p.902-907, 1970.

GRACE, C.; STRIBLEY, D.P. A safer procedure for routine staining of vesicular-arbuscular mycorrhizal fungi. Mycological Research, v.95, p.1160-1162, 1991.

HETRICK, B.A.D.; WILSON, G.W.T.; COX, T.S. Mycorrhizal dependence of modern wheat cultivars and ancestors: a synthesis. Canadian Journal of Botany, v.71, p.512-518, 1993.

JANOS, D.P. Mycorrhiza applications in tropical forestry: are temperate-zone approaches appropriate? In: NG, F.S.P. (Ed.). Trees and mycorrhiza. Kuala Lumpur: Forest Research Institute, 1988. p.133-188.

JANOS, D.P. VA mycorrhizas in humid tropical ecosystems. In: SAFIR, G.R. (Ed.). Ecophysiology of VA mycorrhizal plants. Boca Raton: CRC, 1987. p.107-134.

KOSKE, R.E.; GEMMA, J.N. A modified procedure for staining roots to detect VA mycorrhizas. Mycological Research, v.92, p.486488, 1989.

LOPES, O.M.N.; CARDOSO, E.J.B.N. Efeito do fósforo e da micorriza sobre o crescimento e a absorção de nutrientes por mudas de seringueira (Hevea brasiliensis Muell Arg.). Revista de Ciências Agrárias, v.35, p.41-53, 2001.

LORENZI, H. Árvores brasileiras: manual de identificação e cultivo de plantas arbóreas nativas do Brasil. 3.ed. Nova Odessa: Instituto Plantarum, 2000. 368p.

MELLONI, R.; CARDOSO, E.J.B.N. Quantidade de micélio extraradicular de fungos micorrízicos arbusculares em plantas cítricas. 
II. Comparação entre diferentes espécies cítricas e endófitos. Revista Brasileira de Ciência do Solo, v.23, p.59-67, 1999.

PAULA, M.A.; SIQUEIRA, J.O.; HOSHIKA, E. Crescimento, nutrição e produção de soja inoculada com populações de fungos micorrízicos vesículo-arbusculares. Revista Brasileira de Ciência do Solo, v.14, p.151-156, 1990.

POUYÚ-ROJAS, E. Compatibilidade simbiótica de fungos micorrízicos arbusculares com mudas de espécies arbóreas tropicais. 2002. 90p. Tese (Doutorado) - Universidade Federal de Lavras, Lavras.

SAGGIN JÚNIOR, O.J. Micorrizas arbusculares de espécies arbóreas do Sudeste brasileiro. 1997. 120p. Tese (Doutorado) Universidade Federal de Lavras, Lavras.

SAGGIN JÚNIOR, O.J.; SILVA, E.M.R. Micorriza arbuscular: papel, funcionamento e aplicação da simbiose. Seropédica: Embrapa Agrobiologia, 2002.

SAGGIN JÚNIOR, O.J.; SIQUEIRA, J.O. Avaliação da eficiência simbiótica de fungos endomicorrízicos para o cafeeiro. Revista Brasileira de Ciência do Solo, v.19, p.221-228, 1995.

SAGGIN JÚNIOR, O.J.; SIQUEIRA, J.O. Micorrizas arbusculares em cafeeiro. In: SIQUEIRA, J.O. (Ed.). Avanços em fundamentos e aplicação de micorrizas. Lavras: UFLA: DCS: DCF, 1996. p.203254.

SAGGIN JÚNIOR, O.J.; SIQUEIRA, J.O.; GUIMARÃES, P.T.G.; OLIVEIRA, E. Interação fungos micorrízicos versus superfosfato e seus efeitos no crescimento e teores de nutrientes do cafeeiro em solo não fumigado. Revista Brasileira de Ciência do Solo, v.18, p.27-36, 1994.

SIBINEL, A.H. de M. Resposta da leguminosa Mimosa artemisiana à inoculação com diferentes fungos micorrízicos arbusculares na recuperação de áreas degradadas. 2003. 60p. Dissertação (Mestrado) - Universidade Federal Rural do Rio de Janeiro, Seropédica.

SIEVERDING, E. Vesicular-arbuscular mycorrhiza management in tropical agrosystems. Eschborn: Deutsche Gesellschaft für Technische Zusammenarbeit, 1991. 372p.

SIQUEIRA, J.O.; COLOZZI-FILHO, A. Micorrizas vesículoarbusculares em mudas de cafeeiro. II. Efeito do fósforo no estabelecimento e funcionamento da simbiose. Revista Brasileira de Ciência do Solo, v.10, p.207-211, 1986.

SIQUEIRA, J.O.; COLOZZI-FILHO, A.; SAGGIN JÚNIOR, O.J. Efeitos da infecção de plântulas de cafeeiro com quantidades crescentes de esporos do fungo endomicorrízico Gigaspora margarita. Pesquisa Agropecuária Brasileira, v.29, p.875-883, 1994.

SIQUEIRA, J.O.; SAGGIN JÚNIOR, O.J. Dependency on arbuscular mycorrhizal fungi and responsiveness of some Brazilian native woody species. Mycorrhiza, v.11, p.245-255, 2001.

STRIBLEY, D.P.; TINKER, P.B.; RAYNER, J.H. Relation of internal phosphorus concentration and plant weight in plants infected by vesicular-arbuscular mycorrhizas. New Phytologist, v.86, p.261-266, 1980.

TRINDADE, A.V.; SIQUEIRA, J.O.; ALMEIDA, F.P. Dependência micorrízica de variedades comerciais de mamoeiro. Pesquisa Agropecuária Brasileira, v.36, p.1485-1494, 2001.

Recebido em 21 de dezembro de 2004 e aprovado em 4 de maio de 2005 